\title{
Some remarks on topological structures in the context of fuzzy relational mathematical morphology
}

\author{
Alexander Šostak ${ }^{a, b}$ and Ingrīda Uljane ${ }^{a, b}$ \\ ${ }^{a}$ Institute of Mathematics and CS University of Latvia, \\ aleksandrs.sostaks@lumii.lv, Ingrida Uljane@lumii.lv \\ ${ }^{b}$ University of Latvia, Depatment of Mathematics, \\ aleksandrs.sostaks@lu.lv, ingrida.uljane@lu.lv
}

\begin{abstract}
In this paper, we develop a topological viewpoint on the subject of mathematical morphology. We show that erosion can be interpreted as a certain "remote" interior operator; in its turn dilation can be interpreted as a "remote" closure operator. Two categories are constructed, whose objects are "topological-type" structures obtained by combining operations of erosion and dilation.

Keywords: Mathematical morphology; erosion; dilation; interior; closure; upper and lower image and preimage operators.
\end{abstract}

\section{Introduction}

Mathematical morphology has its origins in geological problems centered in the processes of erosion and dilation. The founders of the mathematical morphology are a civil engineer Jean Serra [27] and a mining engineer Georges Matheron [19]. At the present stage the mathematical morphology can be characterized as a mathematical theory that focuses on studying transformations, especially non-linear, of geometrical objects. Mathematical morphology has applications in different areas of theoretical and applied science, in particular in pattern recognition, image processing, digital topology, etc. Problems related to mathematical morphology were studied by many authors, see e.g. $[4,5,2,3,26,20]$ just to mention some of them Of the various directions where mathematical morphology is being developed, we distinguish two of them: Algebraic mathematical morphology and $L$-fuzzy relational mathematical morphology, see e.g. [20].

The algebraic mathematical morphology takes erosion $\varepsilon$ and dilation $\delta$ as basic concepts. They are defined as mappings on complete lattices $\varepsilon: L_{1} \rightarrow L_{2}$ and $\delta: L_{2} \rightarrow L_{1}$ such that $\varepsilon(\bigwedge A)=\bigwedge_{a \in A} \varepsilon(a) \forall A \subseteq L_{1}$, $\delta(\bigvee B)=\bigvee_{b \in B} \delta(b) \forall B \subseteq L_{2}$, and focuses on the adjunction properties of the pair $(\varepsilon, \delta)$.

The other approach, so called $L$-fuzzy relational mathematical morphology, as its basis has a complete residuated lattice $(L, \vee, \wedge, *, \mapsto)$ where conjunction $*$ is used to define dilation and residuation $\mapsto$ is used to define erosion. We deal only with $L$-fuzzy relational mathematical morphology in this work. Our main interest here concerns topological properties of operators of dilation and erosion.

The paper consists of four sections. In the first section we introduce some special properties of $L$-fuzzy relations used in the sequel. In the second section we consider two kinds of $L$-fuzzy image operators and two kinds of $L$-fuzzy preimage operators. We describe basic properties of these operators, in particular their relations with operators of erosion and dilation. The third section is devoted to topological properties of erosion and dilation and to the categories whose objects are obtained by their combination. In the last section, Conclusion we discuss some directions for the further work.

\section{Operators of erosion and dilation}

Let $L=\left(L, \leq_{L}, \wedge_{L}, \vee_{L}, *\right)$ be a commutative complete lattice monoid with bottom and top elements $0_{L}$ and $1_{L}$ respectively see e.g. [15]. Further, let $\mapsto: L \rightarrow L$ be the residuum related to $*$ via Galois connection, that is $a * b \leq c \Longleftrightarrow a \leq b \mapsto c$ for all $a, b, c \in L$. A complete commutative lattice monoid is called integral if $a * 1_{L}=a$ for all $a \in L$. In this work $L$ is always an integral complete commutative lattice monoid.

Further, let $X$ and $Y$ be sets and $R: X \times Y \rightarrow L$ be an $L$-fuzzy relation.

We will need special properties of $L$-fuzzy relations introduced in the next two definitions:

Definition 1.1 L-fuzzy relation $R$ is called left con- 
nected if $\bigwedge_{y \in Y} \bigvee_{x \in X} R(x, y)=1$. If for every $y \in$ there exists $x \in X$ such that $R(x, y)=1_{L}$, then $R$ is called strongly left connected.

Definition 1.2 $L$-fuzzy relation $R$ is called right connected if $\bigwedge_{x \in X} \bigvee_{y \in Y} R(x, y)=1$. If for every $x \in X$ there exists $y \in Y$ such that $R(x, y)=1_{L}$, then $R$ is called strongly right connected.

Remark 1.3 Properties of left connectedness and strong left connectedness were used in our papers $[28,29]$ under the names of surjectivity and strong surjectivity respectively. Properties of right and strong right connectedness appear in $[28,29]$ under the names of soundness and strong soundness, respectively.

\subsection{Erosion}

Definition 1.4 [20] Given $A \in L^{X}$ its erosion $\varepsilon_{R}(A) \in L^{Y}$ is defined by

$$
\varepsilon_{R}(A)(y)=\bigwedge_{x \in X}(R(x, y) \mapsto A(x)) .
$$

Considering erosion for all $A \in L^{X}$, we get the operator of erosion $\varepsilon_{R}: L^{X} \rightarrow L^{Y}$.

In the next proposition we collect some properties of erosion operator that will be important for us.

Proposition 1.5 (1) Let $a_{X}: X \rightarrow L$ denote a constant function with value $a \in L$. Then $\varepsilon_{R}\left(1_{X}\right)=$ $1_{Y}$ and if $R$ is left connected, then $\varepsilon_{R}\left(a_{X}\right)=a_{Y}$, and in particular $\varepsilon_{R}\left(0_{X}\right)=0_{Y}$

(2) Operator $\varepsilon: L^{X} \rightarrow L^{Y}$ is non-decreasing, that is $A_{1} \leq A_{2} \in L^{X} \Longrightarrow \varepsilon_{R}\left(A_{1}\right) \leq \varepsilon_{R}\left(A_{2}\right)$.

(3) Given a family of L-fuzzy sets $\left\{A_{i} \mid i \in I\right\} \subseteq L^{X}$, we have $\varepsilon_{R}\left(\bigwedge_{i \in I} A_{i}\right)=\bigwedge_{i \in I} \varepsilon_{R}\left(A_{i}\right)$.

Proof. (1) From the definition it is clear that for every $y \in Y$ we have $\varepsilon_{R}\left(a_{X}\right)(y)=\bigwedge_{x \in X}(R(x, y) \mapsto a)$ $=\bigvee_{x \in X} R(x, y) \mapsto a$. In case $a=1_{L}$, we have $\varepsilon_{R}\left(1_{X}\right)(y) \mapsto 1_{L}$ for every $y \in Y$ that is $\varepsilon_{R}\left(1_{X}\right)=$ $1_{Y}$. In its turn, if $R(x, y)$ is left connected, then $\bigvee_{x \in X} R(x, y)=1_{L}$ for every $y \in Y$ and hence $\varepsilon_{R}\left(a_{X}\right)(y)=1_{L} \mapsto a=a$.

(2) Obvious.

(3). Given a family of $L$-fuzzy sets $\left\{A_{i} \mid i \in I\right\}$ and $y \in Y$, we have

$\varepsilon_{R}\left(\bigwedge_{i \in I} A_{i}\right)(y)=\bigwedge_{x \in X}\left(\bigwedge_{i \in I}\left(R(x, y) \mapsto A_{i}(y)\right)\right)=$ $\bigwedge_{i \in I}\left(\bigwedge_{x \in X}\left(R(x, y) \mapsto A_{i}(y)\right)\right)=\bigwedge_{i \in I} \varepsilon_{R}\left(A_{i}\right)(y)$.

\subsection{Dilation}

Definition $1.6[20]$ Given $B \in L^{Y}$, its dilation $\delta_{R}(B) \in L^{X}$ is defined by

$$
\delta_{R}(B)(x)=\bigvee_{y \in Y} R(x, y) * B(y) .
$$

Considering dilation for all $B \in L^{Y}$, we get the operator of dilation $\delta_{R}: L^{Y} \rightarrow L^{X}$.

In the next proposition we collect some properties of dilation operator that will be important for us.

Proposition 1.7 (1) Let $b_{Y}: Y \rightarrow L$ denote a constant function with value $b \in L$. Then $\delta_{R}\left(0_{Y}\right)=$ $0_{X}$ and if $R$ is right connected, then $\delta_{R}\left(b_{Y}\right)=b_{X}$, and in particular $\delta_{R}\left(1_{Y}\right)=1_{X}$

(2) Operator $\delta: L^{Y} \rightarrow L^{X}$ is non-decreasing, that is $B_{1} \leq B_{2} \in L^{Y} \Longrightarrow \delta_{R}\left(B_{1}\right) \leq \delta_{R}\left(B_{2}\right)$.

(3) Given a family of L-fuzzy sets $\left\{B_{i} \mid i \in I\right\} \subseteq L^{Y}$, we have $\delta_{R}\left(\bigvee_{i \in I} A_{i}\right)=\bigvee_{i \in I} \delta_{R}\left(A_{i}\right)$.

Proof (1) For every $x \in X$ we have $\delta_{R}\left(b_{Y}\right)(x)=$

$$
\bigvee_{y \in Y} R(x, y) * b_{Y}(y)=\left(\bigvee_{y \in Y} R(x, y)\right) * b
$$

Hence, $\delta_{R}\left(0_{Y}\right)(x)=0_{L}$ for every $x \in X$, that is $\delta_{R}\left(0_{Y}\right)=0_{X}$. If $R$ is right connected, then $\delta_{R}\left(b_{Y}\right)(x)=b$ for all $x \in X$, that is $\delta_{R}\left(b_{Y}\right)=b_{X}$.

(2) Obvious.

(3) Given a family of $L$-fuzzy sets $\left\{B_{i} \mid i \in I\right\} \subseteq L^{Y}$ and $x \in X$, we have

$$
\begin{gathered}
\delta_{R}\left(\bigvee_{i \in I} B_{i}\right)(x)=\bigvee_{y \in Y}\left(R(x, y) * \bigvee_{i} B_{i}(y)\right)= \\
\bigvee_{y \in Y}\left(\bigvee_{i} R(x, y) * B_{i}(y)\right)= \\
\bigvee_{i \in I}\left(\bigvee_{y \in Y} R(x, y) * B_{i}(y)\right)=\bigvee_{i \in I} \delta_{R}\left(B_{i}\right)(x) .
\end{gathered}
$$

\subsection{Adjunction $\left(\varepsilon_{R}, \delta_{R}\right)$}

It is know that the pair $\left(\varepsilon_{R}, \delta_{R}\right)$ is an adjunction, see e.g. [20]. For completeness we reproduce here the proof of this statement in a form convenient for our exposition.

Given $A \in L^{X}$, and $x \in X$ we have

$$
\begin{gathered}
\delta_{R}\left(\varepsilon_{R}(A)\right)(x)=\bigvee_{y \in Y} R(x, y) * \varepsilon_{R}(A)(y)= \\
\left.\bigvee_{y \in Y} R(x, y) * \bigwedge_{x^{\prime} \in X}\left(R\left(x^{\prime}, y\right)\right) \mapsto A\left(x^{\prime}\right)\right) \leq \\
\left.\bigvee_{y \in Y} R(x, y) *(R(x, y)) \mapsto A(x)\right) \leq A(x)
\end{gathered}
$$


Further, given $B \in L^{Y}$, and $y \in Y$, we have

$$
\begin{gathered}
\varepsilon_{R}\left(\delta_{R}(B)\right)(y)=\bigwedge_{x \in X}\left(R(x, y) \mapsto \delta_{R}(B)(x)\right)= \\
\bigwedge_{x \in X}\left(R(x, y) \mapsto \bigvee_{y^{\prime} \in Y} R\left(x, y^{\prime}\right) * B\left(y^{\prime}\right)\right) \geq \\
\bigwedge_{x \in X}(R(x, y) \mapsto(R(x, y) * B(y))) \geq B(y) .
\end{gathered}
$$

\section{Image and preimage operators on $L$-fuzzy powersets induced by $L$-fuzzy relations}

The subject of this section are, what we call, upper and lower image and preimage operators induced by $L$-fuzzy relation $R: X \times Y \rightarrow L$. As we will see, they are closely related to the operations of erosion and dillation.

\subsection{Upper image and preimage operators}

Let $X, Y$ be sets and $R: X \times Y \rightarrow L$.

Definition 2.1 The upper image of the fuzzy set $A \in$ $L^{X}$ under the L-relation $R: X \times Y \rightarrow L$ is defined by

$$
R^{\rightarrow}(A)(y)=\bigvee_{x} R(x, y) * A(x) \forall A \in L^{X}, \forall y \in Y .
$$

Definition 2.2 The upper preimage of the L-fuzzy set $B \in L^{Y}$ under $L$-fuzzy relation $R: X \times Y \rightarrow L$ is defined by

$$
R^{\leftarrow}(B)(x)=\bigvee_{y} R(x, y) * B(y) \forall B \in L^{Y}, \forall x \in X
$$

Remark 2.3 Thus, dilation $\delta_{R}: L^{Y} \rightarrow L^{X}$ as it is introduced in Definition 1.6, is in fact upper $L$-fuzzy preimage operator $R^{\leftarrow}: L^{Y} \rightarrow L^{X}$.

Remark 2.4 If the fuzzy relation $R$ represents an ordinary function $f: X \rightarrow Y$, then the above definitions reduce respectively, to the definitions of a forward and backward $L$-powerset operators $f^{\rightarrow}$ and $f^{\leftarrow}$, as they are defined by S.E. Rodabaugh [25]. Specifically $f^{\rightarrow}(A)$ is the image $f(A)$ of $A \in L^{X}$ and $f^{\leftarrow}(B)$ is the preimage $f^{-1}(B)$ of $B \in L^{Y}$. The properties of these operators, specifically from the point of view of category theory where thoroughly studied by S.E. Rodabaugh in [25].

In the following proposition we collect basic properties of upper image and preimage operators..

Proposition 2.5 Given sets $X, Y$, let $R: X \times Y \rightarrow L$ be an $L$-fuzzy relation, $\left\{A_{i} \mid i \in I\right\} \subseteq L^{X},\left\{B_{i}: i \in\right.$ $I\} \subseteq L^{Y}$. Then

(1) $R^{\rightarrow}\left(\bigvee_{i \in I}\left(A_{i}\right)\right)=\bigvee_{i \in I} R^{\rightarrow}\left(A_{i}\right)$;
(2) $R \rightarrow\left(\bigwedge_{i \in I}\left(A_{i}\right)\right) \leq \bigwedge_{i \in I} R \rightarrow\left(A_{i}\right)$,

(3) $R^{\leftarrow}\left(\bigvee_{i \in I} B_{i}\right)=\bigvee_{i \in I}\left(R^{\leftarrow} B_{i}\right)$;

(4) $R^{\leftarrow}\left(b_{Y}\right)=b_{X}$ whenever $R$ is right connected.

(5) If $R$ is strongly left connected, then for every $B \in$ $L^{X}$ it holds $R^{\rightarrow}\left(R^{\leftarrow}(B)\right) \geq B$.

(6) If $R$ is left connected, then $R^{\rightarrow}\left(a_{X}\right)=a_{Y}$.

Proof The proofs of the properties (1) - (3) can be found in the literature. We prove the remaining three properties.

(4) $R^{\leftarrow}\left(b_{Y}\right)(y)=\bigvee_{y \in Y} R(x, y) * b_{Y}(y)=$ $\bigvee_{y \in Y} R(x, y) * b=b_{X}$, and hence $R^{\leftarrow}\left(b_{Y}\right)=b_{X}$.

(5) Let $B \in L^{Y}$. Then for every $y \in Y$ $R^{\rightarrow}\left(R^{\leftarrow}(B)\right)(y)=\bigvee_{x} R(x, y) * R^{\leftarrow}(B)(x)$

$=\bigvee_{x} R(x, y) *\left(\bigvee_{y^{\prime}} R\left(x, y^{\prime}\right) * B\left(y^{\prime}\right)\right)$

$\geq \bigvee_{x}(R(x, y) * R(x, y) * B(y)) \geq 1_{L} * B(y)=B(y)$.

(6) $R^{\rightarrow}\left(a_{X}\right)(y)=\bigvee_{x \in X} R(x, y) * a_{X}(x)=$ $\bigvee_{x \in X} R(x, y) * a=a$, and hence $R \rightarrow\left(a_{X}\right)=a_{Y}$.

\subsection{Lower image and preimage operators}

The operators considered in the previous subsection were obtained by applying the idea of Zadeh extension principle [34] and rely on interpretation of operation $*$ as a logical conjunction. On the other hand, here we consider alternative definitions of image and preimage relying on the interpretation of IF-THEN rule as a logical residuation.

Definition 2.6 The lower image of an L-fuzzy set $A \in L^{X}$ is an $L$-fuzzy set $R \Rightarrow(A) \in L^{Y}$ defined by

$$
R^{\Rightarrow}(A)(y)=\bigwedge_{x \in X} R(x, y) \mapsto A(x) .
$$

Remark 2.7 Thus the lower L-fuzzy image operator $R^{\Rightarrow}: L^{X} \rightarrow L^{Y}$ is just the erosion operator $\varepsilon_{R}: L^{X} \rightarrow$ $L^{Y}$

Definition 2.8 The lower preimage of an L-fuzzy set $B \in L^{Y}$ is the $L$-fuzzy set $R^{\Leftarrow}(B) \in L^{X}$ defined by

$$
R^{\Leftarrow}(B)(x)=\bigwedge_{y \in Y} R(x, y) \mapsto B(y) .
$$

Proposition 2.9 If L-fuzzy relation is strongly left connected, then $R^{\Rightarrow}(A) \leq R^{\rightarrow}(A)$ for every $A \in L^{X}$.

Proof Given $y \in Y$, we take $x_{y}$ such that $R\left(x_{y}, y\right)=$ $1_{L}$. Then $\bigwedge_{x \in X} R(x, y) \mapsto A(x) \leq R\left(x_{y}, y\right) \mapsto$ $A\left(x_{y}\right)=A\left(x_{y}\right)$. On the other hand, $\bigvee_{x \in X} R(x, y) *$ $A(x) \geq R\left(x_{y}, y\right) * A\left(x_{y}\right)$. 
Remark 2.10 In case when $L$-fuzzy relation $R$ is realised by an ordinary function $f: X \rightarrow Y$, then $R \Rightarrow(A)$ consists of all $y \in Y$ such that $y=f(x)$ for some $x \in A$ and $y \neq f(x)$ if $x \notin A$. In particular, this property holds if $f$ is injective.

Proposition 2.11 In case $R: X \times Y \rightarrow L$ is strongly right connected, then $R^{\leftarrow} \geq R^{\leftarrow}$

Proof Let $x \in X$ be fixed and let $y_{x} \in Y$ satisfy $R\left(x, y_{x}\right)=1_{L}$. Then $R^{\leftarrow}(B)(x)=\bigvee_{y}(R(x, y) *$ $B(y) \geq R\left(x, y_{x}\right) * B\left(y_{x}\right)=B\left(y_{x}\right)$. In its turn, $R^{\digamma}(B)(x)=\bigwedge_{y}(R(x, y) \mapsto B(y)) \leq R\left(x, y_{x}\right) \rightarrow$ $B\left(y_{x}\right)=B\left(y_{x}\right)$.

Remark 2.12 Note that in case when $L$-fuzzy relation $R$ is realized by an ordinary function $f$, then $R^{\leftarrow}(B)=R^{\leftarrow}(B)=f^{-1}(B)$, that is the preimage of $B$ under function $f$. Strong right connectedness in this case just means that the function $f$ is surjective.

In the next proposition we collect some properties of lower image and preimage operators.

Proposition 2.13 Let $R: X \times Y \rightarrow L$ be an L-fuzzy relation, $A \in L^{X}, B \in L^{Y},\left\{A_{i} \mid i \in I\right\} \subseteq L^{X}$ and $\left\{B_{i} \mid i \in I\right\} \subseteq L^{Y}$. Then

(1) If $A_{1} \leq A_{2}$ then $R \Rightarrow\left(A_{1}\right) \leq R \Rightarrow\left(A_{2}\right)$;

(2) If $B_{1} \leq B_{2}$ then $R^{\Leftarrow}\left(B_{1}\right) \leq R^{\Leftarrow}\left(B_{2}\right)$;

(3) $R^{\Rightarrow}\left(\bigwedge_{i \in I} A_{i}\right)=\bigwedge_{i \in I} R^{\Rightarrow}\left(A_{i}\right)$

(4) $R^{\Leftarrow}\left(\bigwedge_{i \in I} B_{i}\right)=\bigwedge_{i \in I} R^{\Leftarrow}\left(B_{i}\right)$

(5) If $R$ is left connected, then $R^{\Rightarrow}\left(a_{X}\right)=a_{Y}$

(6) If $R$ is right connected $R^{\Leftarrow}\left(b_{Y}\right)=b_{Y}$

Proof The proof of the properties (1) - (4) can be found in the literature. We prove the remaining two properties.

To prove (5) we fix $y \in Y$ and, taking into account that $R$ is left connected, are reasoning as follows: $R \Rightarrow\left(a_{X}\right)(y)=\bigwedge_{x \in X} R(x, y) \mapsto a_{X}(x)=1_{L} \mapsto a=$ $a_{Y}(y)$. The proof of $(6)$ is similar to the proof of (5) and omitted.

\section{Topological structures in the context of mathematical morphology}

\subsection{Some remarks concerning terminology}

Before we start to consider the operators of erosion and dilation from the topological point of view, we have to specify terminology to which we stick here. Of several equivalent approaches to a definition of a topology, we use here the interior-closure approach. Besides, since we are working with $L$-fuzzy sets, we speak about $L$ fuzzy interior and $L$-fuzzy closure operators. However, we give here also references to their classic prototypes.

Recall that an $L$-fuzzy interior operator on a set $X$ is a mapping int $: L^{X} \rightarrow L^{X}$ such that

(1) $\operatorname{int}\left(1_{X}\right)=1_{L}$;

(2) $\operatorname{int}(A) \leq A$ for every $A \in L^{X}$;

(3) $\operatorname{int}\left(A_{1} \wedge A_{2}\right)=\operatorname{int}\left(A_{1}\right) \wedge \operatorname{int}\left(A_{1}\right) \forall A_{1}, A_{2} \in L^{X}$,

(4) $\operatorname{int}(\operatorname{int}(A))=\operatorname{int}(\mathrm{A})$ for every $A \in L^{X}$.

see e.g. [11], [17], [31]. In case int satisfies a stronger version of axiom (1)

$\left(1^{\prime}\right) \operatorname{int}\left(a_{X}\right)=a_{L}$ for each constant $a \in L$,

int is called stratified, see e.g. [17], [16], but in case int satisfies a stronger version of axiom (3)

$\left(3^{\prime}\right) \operatorname{int}\left(\bigwedge_{i \in I} A_{i}\right)=\bigwedge_{i \in I} \operatorname{int}\left(A_{i}\right)$,

operator int is called Alexandroff L-fuzzy interior. [1]. In case the axiom (3) is omitted, we come to the concept of an $L$-fuzzy pre-interior operator (stratified and Alexandroff pre-interior operator, respectively.)

An $L$-fuzzy (pre-)interior operator gives rise to an $L$ fuzzy (pre-)topology $T$ by setting

$$
T=\left\{A \in L^{X} \mid \operatorname{int}(A)=A\right\} .
$$

An $L$-fuzzy closure operator on a set $X$ is a mapping $\operatorname{cl}: L^{X} \rightarrow L^{X}$ such that

(1) $\operatorname{cl}\left(0_{X}\right)=0_{L}$;

(2) $\operatorname{cl}(A) \geq A$ for every $A \in L^{X}$

(3) $\operatorname{cl}\left(A_{1} \vee A_{2}\right)=\operatorname{cl}\left(A_{1}\right) \vee \operatorname{cl}\left(A_{1}\right) \forall A_{1}, A_{2} \in L^{X}$,

(4) $\operatorname{cl}(\operatorname{cl}(A))=\operatorname{cl}\left(\right.$ A) for every $A \in L^{X}$

see e.g. [11], [17], [31]. In case cl satisfies a stronger version of axiom ( 1 )

(1') $\operatorname{cl}\left(a_{X}\right)=a_{L}$ for each constant $a \in L$,

cl is called co-stratified, see e.g. [16], but in case cl satisfies a stronger version of axiom $(3 \mathrm{cl})$

$\left(3^{\prime}\right) \quad \operatorname{cl}\left(\bigvee_{i \in I} A_{i}\right)=\bigvee_{i \in I} \operatorname{cl}\left(A_{i}\right)$, 
operator cl is called Alexandroff $L$-fuzzy closure, see $[1,7]$. In case the axiom $(3 \mathrm{cl})$ is omitted, we come to the concept of $L$-fuzzy pre-closure operator (stratified and Alexandroff pre-closure resp.)

An $L$-fuzzy (pre-)closure operator gives rise to an $L$ fuzzy (pre-)co-topology $T$ by setting

$$
C=\left\{A \in L^{X} \mid \operatorname{cl}(A)=A\right\} .
$$

In case when the lattice is endowed with an order reversing involution ${ }^{c}: L \rightarrow L$ the closure and interior operators are usually considered as a pair mutually related operators connected by the following equalities

$$
\operatorname{cl}\left(A^{c}\right)=(\operatorname{int}(A))^{c} ; \operatorname{int}\left(A^{c}\right)=(\operatorname{cl}(A))^{c} \forall A \in L^{X},
$$

and hence $\mathrm{cl}: L^{X} \rightarrow L^{X}$ and int $: L^{X} \rightarrow L^{X}$ present equivalent approaches to an $L$-fuzzy (pre)-topology. However, there are situations when either the lattice $L$ does not have a natural order reversing involution or interior and closure operators base on different information in their definitions. In this case the term a (fuzzy) di(pre)topology is used, see e.g. [6]. This is just the case in our situation: the $L$-fuzzy pre-interior and $L$-fuzzy pre-closure operators which appear in our work will be generally independent.

\subsection{Pair $\left(\varepsilon_{R}, \delta_{R^{-1}}\right)$ as an $L$-fuzzy di-pretopology transformed from $L^{X}$ to $L^{Y}$}

Let $R: X \times Y \rightarrow L$ be an $L$-fuzzy relation interpreted as a relation from $X$ to $Y$ and let $R^{-1}: Y \times X \rightarrow L$ defined by $R^{-1}(y, x)=R(x, y)$ and hence interpreted as a relation from $Y$ to $X$.

The properties of erosion operator $\varepsilon_{R}$ collected in Proposition 1.5 remind the properties of a stratified Alexandroff fuzzy pre-interior operator defined on the $L$-powerset $L^{X}$ but taking values in $L^{Y}$. On the other hand, the properties of the operator of dilation $\delta_{R}$ collected in Proposition 2.3 and reformulated for $\delta_{R^{-1}}$ remind the properties of the stratified Alexandroff fuzzy pre-closure operator defined on the $L$-powerset $L^{X}$ and taking values in $L^{Y}$. From the definitions it is clear that $\varepsilon_{R}(A)=R^{\Rightarrow}(A)$, (see Remark 2.7) and $\delta_{R^{-1}}(A)=R^{\rightarrow}(A)$ (see Remark 2.3). Now, referring to Proposition 2.9 we know that if $R$ is strongly right connected, then $R^{\Rightarrow}(A) \leq R^{\rightarrow}(A)$. These observations lead us to the interpretation of $\varepsilon_{R}(A)$ as the $L$ fuzzy pre-interior of $A \in L^{X}$ transferred to $L^{Y}$ and $\delta_{R^{-1}}(A)$ as the $L$-fuzzy pre-closure of $A$ transferred to $L^{Y}$. In the result we interpret the pair $\left(\varepsilon_{R}, \delta_{R^{-1}}\right)$ as an Alexandroff $L$-fuzzy di-pretopology transformed from $L^{X}$ to $L^{Y}$.

\subsection{Pair $\left(\varepsilon_{R^{-1}}, \delta_{R}\right)$ as an $L$-fuzzy di-pretopology transformed from $L^{Y}$ to $L^{X}$}

As above, let $R: X \times Y \rightarrow L$ be an $L$-fuzzy relation and let $R^{-1}: Y \times X \rightarrow L$ defined by $R^{-1}(y, x)=$ $R(x, y)$ and hence interpreted as relation from $Y$ to $X$. The properties of dilation operator $\delta_{R}$ collected in Proposition 1.7 remind the properties of an Alexandroff pre-closure operator defined on the $L$-powerset $L^{Y}$ but taking values in $L^{X}$. On the other hand the properties of operator $\varepsilon_{R}$ collected in Proposition 1.5 and reformulated for $\varepsilon_{R^{-1}}$ remind the properties of Alexandroff $L$-fuzzy fuzzy closure operator defined on the $L$-powerset $L^{Y}$ but taking values in $L^{X}$.. Reasoning in a similar way as in the previous subsection, we notice that $\varepsilon_{R^{-1}}(B)=R^{\leftarrow}(B)$, and $\delta_{R}(B)=R^{\leftarrow}(B)$ for every $B \in L^{Y}$. Further, referring to Proposition 2.11 , we know that if $R$ is strongly left connected, then $R^{\Leftarrow}(B) \leq R^{\leftarrow}(B)$. These observations lead us to the interpretation of $\varepsilon_{R^{-1}}(B)$ as the Alexandroff $L$-fuzzy pre-interior of $B \in L^{Y}$ transferred to $L^{X}$ and $\delta_{R}(B)$ as the Alexandroff $L$-fuzzy pre-closure of $B \in L^{Y}$ transferred to $L^{X}$. In the result we interpret the pair $\left(\varepsilon_{R^{-1}}, \delta_{R}\right)$ as an $L$-fuzzy di-pretopology transformed from $L^{Y}$ to $L^{X}$.

\subsection{Remark: the case $X=Y$}

In case when $X=Y$ and $R: X \times X \rightarrow L$ is a reflexive transitive $L$-fuzzy relation, operator $\varepsilon_{R}: L^{X} \rightarrow L^{X}$ is the $L$-fuzzy closure operator and operator $\delta_{R}: L^{X} \rightarrow$ $L^{X}$ is an $L$-fuzzy interior operator. This situation was studied from different points of view by many authors, see e.g. $[9,21,12,14,23,24]$ et al. However in all these works the $L$-fuzzy relation was on the same set $X$, that is $R: X \times X \rightarrow L$ and hence the image and preimage operators where of the form $R^{\rightarrow}: L^{X} \rightarrow L^{X}, R^{\leftarrow}: L^{X} \rightarrow L^{X}, R^{\Rightarrow}: L^{X} \rightarrow L^{X}$, $R^{\Leftarrow}: L^{X} \rightarrow L^{X}$. On the other hand the context of the mathematical morphology focuses on the situation when the image and preimage can take their values in different universies, that is when the obtained image and preimage operators are of the form $R^{\rightarrow}: L^{X} \rightarrow L^{Y}, R^{\leftarrow}: L^{Y} \rightarrow L^{X}, R^{\Rightarrow}: L^{X} \rightarrow L^{Y}$, $R^{\Leftarrow}: L^{Y} \rightarrow L^{X}$ that is the subject of this paper.

Note also that in this case, according to the terminology accepted in mathematical morphology, composition $\varepsilon_{R} \circ \delta_{R}: L^{X} \rightarrow L^{X}$ is called an opening operator and composition $\delta_{R} \circ \varepsilon_{R}: L^{X} \rightarrow L^{X}$ is a closing operator.

\subsection{Category of $\left(\varepsilon, \delta^{-1}\right)$-spaces}

Definition $3.1 A n \quad\left(\varepsilon, \delta^{-1}\right)$-space is the tuple $\left(X, Y, L, R, \varepsilon_{R}, \delta_{R^{-1}}\right)$ where $X, Y$ are sets, $L$ is a fixed 
integral complete lattice monoid, $R: X \times Y \rightarrow L$ is an $L$-fuzzy relation and $\varepsilon_{R}, \delta_{R^{-1}}$ are erosion and dilation operators.

To view $\left(\varepsilon, \delta^{-1}\right)$-spaces as a category we must specify its morphisms. We do it in the next definition justified by the topological interpretation of the pairs $\left(\varepsilon_{R}, \delta_{R^{-1}}\right)$ given above:

Definition 3.2 A continuous transformation from an $\left(\varepsilon, \delta^{-1}\right)$-space $\left(X_{1}, Y_{1}, L, R_{1}, \varepsilon_{R_{1}}, \delta_{R_{1}^{-1}}\right)$ to an $\left(\varepsilon, \delta^{-1}\right)$ space $\left(X_{2}, Y_{2}, L, R_{2}, \varepsilon_{R_{2}}, \delta_{R_{2}^{-1}}\right)$, is a pair of mappings $(\varphi, \psi)$ where $\varphi: X_{1} \rightarrow X_{2}, \psi: Y_{1} \rightarrow Y_{2}$ satsfying the following conditions:

(1) $\varepsilon_{R_{2}}(\varphi(A)) \leq \psi\left(\varepsilon_{R_{1}}(A)\right) \forall A \in L^{X_{1}}$

(2) $\delta_{R_{2}^{-1}}(\varphi(A)) \leq \psi\left(\delta_{R_{1}^{-1}}(A)\right) \forall A \in L^{X_{1}}$.

Given three $\left(\varepsilon, \delta^{-1}\right)$-spaces and continuous transformations

$(\varphi, \psi)$ :

$\left(X_{1}, Y_{1}, L, R_{1}, \varepsilon_{R_{1}}, \delta_{R_{1}^{-1}}\right) \rightarrow\left(X_{2}, Y_{2}, L, R_{2}, \varepsilon_{R_{2}}, \delta_{R_{2}^{-1}}\right)$, $\left(\varphi^{\prime}, \psi^{\prime}\right)$ :

$\left(X_{2}, Y_{2}, L, R_{2}, \varepsilon_{R_{2}}, \delta_{R_{2}^{-1}}\right) \rightarrow\left(X_{3}, Y_{3}, L, R_{3}, \varepsilon_{R_{3}}, \delta_{R_{3}^{-1}}\right)$ we define their composition as $\left(\varphi^{\prime} \circ \varphi, \psi^{\prime} \circ \psi\right)$ :

$\left(X_{1}, Y_{1}, L, R_{1}, \varepsilon_{R_{1}}, \delta_{R_{1}^{-1}}\right) \rightarrow\left(X_{3}, Y_{3}, L, R_{3}, \varepsilon_{R_{3}}, \delta_{R_{3}^{-1}}\right)$.

The proof of the next proposition is straightforward and omitted:

Proposition $3.3\left(\varepsilon, \delta^{-1}\right)$-spaces and their continuous transformations with composition defined above form a category.

\subsection{Category of $\left(\varepsilon^{-1}, \delta\right)$-spaces}

Definition 3.4 An $\left(\varepsilon^{-1}, \delta\right)$-space is a tuple $\left.\left(X, Y, L, R, \varepsilon_{R^{-1}}, \delta_{R}\right)\right)$ where $X, Y$ are sets, $L$ is a fixed integral comutative complete lattice monoid, $R: X \times Y \rightarrow L$ is an L-fuzzy relation and $\varepsilon_{R^{-1}}, \delta_{R}$ are erosion and dilation operators.

To view $\left(\varepsilon_{R^{-1}}, \delta_{R}\right)$-spaces as a category we must specify its morphisms. We do it in the next definition justified by the topological interpretation of the pairs $\left(\varepsilon^{-1}, \delta\right)$-spaces given above:

Definition 3.5 A continuous transformation from $a$ space $\left.\left(X_{1}, Y_{1}, L, R_{1}, \varepsilon_{R_{1}^{-1}}, \delta_{R_{1}}\right)\right)$ to a space $\left(X_{2}, Y_{2}, L, R_{2}, \varepsilon_{R_{2}^{-1}}, \delta_{R_{2}}\right)$, is a pair of mappings $(\varphi, \psi)$ where $\varphi: X_{1} \rightarrow X_{2}, \psi: Y_{1} \rightarrow Y_{2}$ satisfying the following conditions:

(1) $\left.\varphi\left(\varepsilon_{R_{1}^{-1}}(B)\right)\right) \leq \varepsilon_{R_{2}^{-1}}(\psi(B)) \forall B \in L^{Y_{1}}$;
(2) $\varphi\left(\delta_{R_{1}}(B)\right) \leq \delta_{R_{2}}(\psi(B)) \forall B \in L^{Y_{1}}$.

Composition of continuous transformations is defined similar as it was done in case of the category of $\left(\varepsilon, \delta^{-1}\right)$-spaces.

The proof of the following proposition is straightforward and therefore omitted:

Proposition $3.6\left(\varepsilon^{-1}, \delta\right)$-spaces and their continuous transformations form a category.

\section{Conclusions}

In this paper, we have developed a certain topological interpretation of basic concepts of fuzzy relational mathematical morphology. We have showed that the erosion operator $\varepsilon_{R}: L^{X} \times L^{Y}$ induced by an $L$ fuzzy relation $R: X \times Y \rightarrow L$ can be realized as a certain $L$-fuzzy pre-interior operator assigning to an $L$-fuzzy subset $A \in L^{X}$ its "transferred" $L$-fuzzy pre-interior $\varepsilon_{R}(A) \in L^{Y}$, while the dilation operator $\delta_{R^{-1}}: L^{X} \rightarrow L^{Y}$ induced by the inverse $L$-fuzzy relation $R^{-1}: Y \times X \rightarrow L$ can be realized as a certain $L$-fuzzy pre-closure operator assigning to an $L$ fuzzy subset $A \in L^{X}$ its "transferred" $L$-fuzzy preinterior $\varepsilon_{R}(A) \in L^{Y}$. In its turn, the erosion operator $\varepsilon_{R^{-1}}: L^{Y} \rightarrow L^{X}$ and the dilation operator $\delta_{R}: L^{Y} \rightarrow L^{X}$ can be realized as certain $L$-fuzzy preinterior and $L$-fuzzy pre-closure operators defined on $L^{Y}$ and taking values in $L^{X}$. The operators of erosion and dilation were compared with the upper and lower images and preimages operators induced by $L$ fuzzy relations. We have initiated also the study of the topological-type structures obtained here from the categorical point of view.

Concerning the prospectives for the future work, as the most chalenging we see the following.

- In this paper we just sketched the ideas for the categorical approach to the pre-topological structures in the context of fuzzy relational mathematical morphology. In future, we plan to develop a "full-boded" categorical approach to such structures.

- The pairs $\left(\varepsilon_{R}, \delta_{R^{-1}}\right)$ and $\left(\varepsilon_{R^{-1}}, \delta_{R}\right)$ were used here to develop a certain "topological viewpoint" on the operators of erosion and dilation. However, probably even more interesting especially from the point of possible application will be to apply these pairs for description of certain "remote" fuzzy rough approximation models (cf [22], [8], [10], [33]). In particular, it could be useful in the study of big volumes of transformed data. 
- In [20] the authors have extended the subject of mathematical morphology for adjoint triples. We guess that this theory can have important relations with the so called "non-commutative" topology that we are going to explore in future.

\section{Acknowledgements}

The authors are thankful for the partial financial support from the project No Lzp-2018/2-0338 'Development of fuzzy logic based technologies for risk assessment by means of relational-grounded aggregation' by the Latvian Council of Science.

The first named author expresses his gratitude to Manuel Ojeda-Aciego for drawing his attention to the topic of Mathematical Morphology and sending the preprint version of the paper [20].

Both authors are thankfull to the two anonymous referees for reading the paper carefully and making some critical comments.

\section{References}

[1] Alexandroff, P: Diskrete Räume. Mat. Sbornik 2 (1937), 501-518.

[2] De Baets, B, Kerre, E.E., Gupta, M.: The fundamentals of fuzzy mathematical morphology Part I: basic concepts. International J. of General Systems 23 (1995), 155-171.

[3] De Baets, B, De, Kerre, E.E., Gupta, M.: The fundamentals of fuzzy mathematical morphology Part II: idempotence, convexity and cecomposition. International J. of General Systems 23 (1995), 307-322.

[4] Bloch, I.: Duality vs. adjunction for fuzzy mathematical morhology, Fuzzy Sets and Syst. 160 (2009), 1858 - 1867.

[5] Bloch, I., Maitre, H. Fuzzy mathematical morphology: a comparative study. Pattern Recogn. 28 (1995), 1341-1387.

[6] Brown, L.M., Ertürk, R., Dost Ş. : Ditopological texture spaces and fuzzy topology, I. Basic concepts. Fuzzy Sets and Syst. 110 (2000), 227-236.

[7] Chen, P., Zhang, D. : Alexandroff $L$-cotopological spaces. Fuzzy Sets and Systems 161 (2010) 2505 -2525 .

[8] Dubois, D., Prade, H.: Rough fuzzy sets and fuzzy rough sets. Intern. J. General Systems $\mathbf{1 7}$ (1990), 191-209.
[9] Fang Jingming I-fuzzy Alexandrov topologies and specialization orders, Fuzzy Sets and Systems, 158 (2007), 2359-2374.

[10] Elıkins, A., Šostak, A., Uljjane, I.: On a category of extensional fuzzy rough approximation operators. In: Communication in Computer Inform. Sci., vol 611 (2016).

[11] Engelking, R.: General topology. PWN, Warszawa, 1985.

[12] J. Hao, Q. Li The relation between L-fuzzy rough sets and L-topology, Fuzzy Sets and Systems, 178 (2011) 74-83.

[13] Hájek, P.: Methamatimatics of Fuzzy Logic, Kluwer Acad. Publ., 1998.

[14] Han, S-E., Šostak, A.: On the measure of Mrough approximation of $L$-fuzzy sets. Soft Computing 22 (2018), 2843-2855.

[15] Höhle, U.: M-valued sets and sheaves over integral commutative cl-monoids, Chapter 2 In: S.E. Rodabaugh, E.P. Klement, U. Höhle (eds.) Applications of Category Theory to Fuzzy Sets. Kluwer Acad. Publ. (1992), pp. 33-73.

[16] Höhle, U., Šostak, A.: Axiomatic foundations of fixed-based fuzzy topology, In: Höhle, U., Rodabaugh, S. (eds.) Mathematics of Fuzzy Sets: Logic, Topology and Measure Theory, pp. 123272 Kluwer Acad. Publ. (1999).

[17] Liu, Yingming, Luo, Maokang, Fuzzy Topology Advances in Fuzzy Systems - Applications and Topology. World Scientific. Singapore, New Jersey, London, Hong Kong, 1997.

[18] Nachtegael, M., Sussner, P., Melange, T., Kerre, E.E. : On the role of complete lattices in mathgematical morphology: from tool to uncertainty model. Information Sciences 181 (2011) 19711988.

[19] Matheron, G. Random sets and Integral Geometry, Willy, 1975.

[20] Madrid, N., Ojeda-Aciego, M., Medina, J., Perfilieva, I.: $L$-fuzzy relational mathematical morphology based on adjoint triples. Information Sciences 474 (2019), 75-89.

[21] J.S. Mi, B.Q. Hu Topological and lattice structure of L-fuzzy rough sets determined by upper and lower sets, Information Sciences 218 (2013), 194-204.

[22] Pawlak, Z.: Rough sets. Intern. J. of Computer and Inform. Sci. 11 (1982), 341-356. 
[23] K. Qin, Z. Pei, On the topological properties of fuzzy rough sets, Fuzzy Sets and Systems 151 (2005), 601-613.

[24] K. Qin, Z. Pei, Generalized rough sets based on reflexive and transitive relations, Information Sciences 178 (2008), 4138-4141.

[25] Rodabaugh, S.E.: Powerset operator based foundations for poslat fuzzy set theories and topologies, Quaest. Math. 20 (1997), 463-530.

[26] Ronse, C.: Why mathematical morphology needs complete lattices, Signal Processing 21 (1990), 129-154.

[27] Serra, J. : Image analysis and mathematical morphology, I. Academic Press, 1982.

[28] Šostak, A. Uljane, I.: M-bornologies on L-valued sets, Advances in Intelligent Systems and Computing, vol 643, 450-462.

[29] Šostak, A. Uljane, I.: Bornological structures on many-valued sets, RAD HAZU Matematiçke Znanosti 21 (2017) 145-170.

[30] Šostak, A.: Two decades of fuzzy topology: Basic ideas, notions and results, Russian Math. Surveys, 44 (1989), 125-186

[31] Šostak, A.: Basic structures of fuzzy topology, J. Math. Sci. 78 (1996), 662-701.

[32] Šostak, A., Elkkins, A.: $L M$-valued equalities, $L M$-rough approximation operators and $M L$ graded ditopologies. Hacettepe J. Math. Stat. 46 (2017), 15-32.

[33] Yao Y., She Y.: Rough set models in multigranulationl spaces. Inform. Sci. 327 (2016), 40-56.

[34] Zadeh, L.A.: The concept of linguistic variable and its application to approximate reasoning. Inform. Sci. 8 (1975), 193-249, 301-357. 